Research Article

\title{
Event-by-Event Particle Ratio Fluctuations at LHC Energies
}

\author{
Shaista Khan (D), Bushra Ali $\mathbb{D}^{D}$, Anuj Chandra $(\mathbb{D}$, and Shakeel Ahmad
}

Department of Physics, Aligarh Muslim University, Aligarh 202002, India

Correspondence should be addressed to Shaista Khan; shaista.khan@cern.ch

Received 1 January 2021; Revised 4 July 2021; Accepted 10 August 2021; Published 12 October 2021

Academic Editor: Raghunath Sahoo

Copyright (C) 2021 Shaista Khan et al. This is an open access article distributed under the Creative Commons Attribution License, which permits unrestricted use, distribution, and reproduction in any medium, provided the original work is properly cited. The publication of this article was funded by $\mathrm{SCOAP}^{3}$.

\begin{abstract}
A Monte Carlo study of identified particle ratio fluctuations at LHC energies is carried out in the framework of HIJING model using the fluctuation variable $v_{\text {dyn }}$. The simulated events for $\mathrm{Pb}-\mathrm{Pb}$ collisions at $\sqrt{s}_{N N}=2.76$ and $5.02 \mathrm{TeV}$ and Xe-Xe collisions at $\sqrt{s}_{N N}=5.44 \mathrm{TeV}$ are analyzed. From this study, it is observed that the values of $[\pi, K],[p, K]$, and $[\pi, p]$ follow the similar trends of energy dependence as observed in the most central collision data by NA49, STAR, and ALICE experiments. It is also observed that $v_{\mathrm{dyn}}$ for all the three combinations of particles for semicentral and central collisions, the model predicted values of $v_{\text {dyn }}[A, B]$ for $\mathrm{Pb}-\mathrm{Pb}$ collisions at $\sqrt{s}_{N N}=2.76 \mathrm{TeV}$ agree fairly well with those observed in the ALICE experiment. For peripheral collisions, however, the model predicted values of $v_{\text {dyn }}[\pi, K]$ are somewhat smaller, whereas for $[p, K]$ and $[\pi, p]$ it predicts larger values as compared to the corresponding experimental values. The possible reasons for the observed differences are discussed. The $v_{\text {dyn }}$ values scaled with charged particle density when plotted against $\left\langle N_{\text {part }}\right\rangle$ exhibit a flat behaviour, as expected from the independent particle emission sources. For $[p, K]$ and $[\pi, p]$ combinations, a departure from the flat trend is, however, observed in central collisions in the case of low $p_{T}$ window when the effect of jet quenching or resonances is considered. Furthermore, the study of $v_{\text {dyn }}[A, B]$ dependence on particle density for various collision systems (including proton-proton collisions) suggests that at LHC energies $v_{\text {dyn }}$ values for a given particle pair are simply a function of charged particle density, irrespective of system size, beam energy, and collision centrality.
\end{abstract}

\section{Introduction}

Fluctuations associated to a physical quantity measured in an experiment, in general, depend on the property of the system and are expected to provide useful clue about the nature of the system under study [1-3]. As regards the heavy-ion (AA) collisions, the system created is assumed to be a hot and dense fireball of hot partonic and (or) hadronic matter $[1,2]$. One of the main aims of studying AA collisions at relativistic energies is to search for the existence of partonic matter in the early stage of the created fireball. Fluctuations associated to a thermal system are supposed to be related to various susceptibilities $[1,2,4]$ and would serve as an indicator of the possible phase transition. Moreover, the presence of large event-by-event (ebe) fluctuations, if observed, might be signal for the presence of distinct classes of events, one with and one without QGP formation [5-7]. Therefore, the search for the phase transition from hadronic matter to QGP still remains a topic of interest of high-energy physicists [8-10]. Correlations and ebe fluctuations of dynamical nature are believed to be associated with the critical phenomena of phase transition and their studies would lead to the local and global differences between the events produced under similar initial conditions [11].

ebe fluctuations in hadronic and heavy-ion collisions have been investigated at widely different energies using several different approaches, for example, normalized factorial moments [12-15], multifractals $[16,17], k$-order rapidity spacing [18-20], erraticity [21-23], and intensive and strongly intensive quantities (defined in terms of multiplicity, transverse momentum, $p_{T}$, etc.) [24-26]. Furthermore, ebe fluctuations in conserved quantities like strangeness, 
baryon number, and electric charge have emerged as new tools to estimate the degree of equilibration and criticality of the measured systems [27-31]. The dynamical net charge fluctuations have been investigated by STAR and ALICE experiments $[28,29]$ in terms of variable $v_{\text {dyn }}[32]$, which is an excellent probe because of its robustness against detector efficiency losses [29]. The other measures of the net charge fluctuations, like the variance of charge $V(Q)$, variance of charge ratio $V(R)$, and the $D$-measure $[29,31$, $33,34]$, are prone to the measurement conditions $[32,35]$.

It has, however, been pointed out [36] that large systematic uncertainties, like volume fluctuations due to impact parameter variations, are associated in such measurements, while the multiplicity ratio fluctuations are sensitive to the density fluctuations instead of volume fluctuations [37]. Thus, the variable $v_{\text {dyn }}$, defined by considering the particle species pair, rather than defining it in terms of combinations of like and unlike charges, has been used as a tool to probe the properties of QGP $[33,38]$. Since it is speculated that the phase transition, if occurs, would result in increase and divergence of fluctuations and could be related to ebe fluctuations of a suitably chosen observable. An enhanced fluctuation in the particle ratio is expected during a phase transition at critical point $(\mathrm{CP})$. For example, $[\pi, K],[p, K]$, and $[\pi, p]$ fluctuations could be related to baryon number fluctuations, strangeness fluctuations, and baryon-strangeness correlations $[38,39]$.

Particle ratio fluctuations in AA collisions have been addressed in a number of studies, e.g., NA49 experiment in $\mathrm{Pb}-\mathrm{Pb}$ collisions at $\mathrm{E}_{\mathrm{lab}}=20-158 \mathrm{~A} \mathrm{GeV} \mathrm{[40],} \mathrm{STAR} \mathrm{exper-}$ iment in Au-Au collisions at $\sqrt{s}_{N N}=7.7$ to $200 \mathrm{GeV}$ [41], $\mathrm{Cu}-\mathrm{Cu}$ collisions at $\sqrt{s}_{N N}=22.4,62.4$, and $200 \mathrm{GeV}$ [42], and several others $[33,36,38]$. At LHC energies, the particle ratio fluctuations have been investigated by the ALICE experiment at $\sqrt{s}_{N N}=2.76 \mathrm{TeV}$ only [43-45]. It has been reported [43] that $v_{\text {dyn }}$ for $[\pi, K]$ and $[p, K]$ combinations acquires positive values irrespective of the centrality class, whereas, for $[\pi, p]$ combination, the variable changes sign from positive to negative toward more peripheral collisions, indicating the difference in the production mechanisms involved of these pairs. The observed trend of energy dependence of $v_{\text {dyn }}$ with beam energy [43] suggests that the production dynamics changes significantly from that reported at lower energies. It has also been pointed out [43] that further investigations involving fluctuations with charge and species specific pairs are carried out to characterize the production dynamics and understand the observed sign changes. It was, therefore, considered to undertake the study of particle ratio fluctuations by analyzing the data on $\mathrm{Pb}-\mathrm{Pb}$ collisions at $\sqrt{s}_{N N}=2.76$ and $5.02 \mathrm{TeV}$ and Xe-Xe collisions at $\sqrt{s}_{N N}=5.44 \mathrm{TeV}$ in the framework of HIJING model. Using the HIJING, the effect of jet quenching and resonance production can also be looked into.

\section{Formalism}

The particle ratio fluctuations may be studied in terms of the yields of the ratio of particle types $A$ and $B$. The particle ratio
$A / B$ is estimated by counting the particle types $A$ and $B$ produced in each event. Using the relative widths of the particle ratio distributions of the data and the corresponding mixed events the observable $\sigma_{\mathrm{dyn}}$ is defined as $[38,46]$

$$
\sigma_{\text {dyn }}=\operatorname{sgn}\left(\sigma_{\text {data }}^{2}-\sigma_{\text {mixed }}^{2}\right) \sqrt{\left|\sigma_{\text {data }}^{2}-\sigma_{\text {mixed }}^{2}\right|},
$$

where $\sigma_{\text {data }}$ and $\sigma_{\text {mixed }}$, respectively, denote the relative widths (standard deviation/mean) of the ratio $A / B$ for the data and mixed events. Yet another variable $v_{\mathrm{dyn}}$, which is commonly accepted for studying the particle ratio fluctuations, has been proposed [32]. $v_{\mathrm{dyn}}[A, B]$ quantifies the deviation of the fluctuations in the number of particle species $A$ and $B$ from that expected from Poissonian statistics [46]. This variable does not involve particle ratios directly but is related to $\sigma_{\mathrm{dyn}}$ as $\sigma_{\mathrm{dyn}}^{2} \approx v_{\mathrm{dyn}}[A, B][42,46]$.

$v_{\text {dyn }}[A, B]$ is defined as $[38,43-46]$

$$
v_{\mathrm{dyn}}[A, B]=\frac{\left\langle N_{A}\left(N_{A}-1\right)\right\rangle}{\left\langle N_{A}\right\rangle^{2}}+\frac{\left\langle N_{B}\left(N_{B}-1\right)\right\rangle}{\left\langle N_{B}\right\rangle^{2}}-2 \frac{\left\langle N_{A} N_{B}\right\rangle}{\left\langle N_{A}\right\rangle\left\langle N_{B}\right\rangle},
$$

where $\left\langle N_{A}\right\rangle$ and $\left\langle N_{B}\right\rangle$, respectively, denote the event multiplicities of particle types $A$ and $B$ within the given kinematical limits, while the quantities within $\langle\cdots\rangle$ represent their mean values. It should be mentioned here that the particle type $A$ or $B$ includes the particle and its antiparticle. $v_{\text {dyn }}[A, B]$ basically contrasts the relative strengths of fluctuations of particle types $A$ and $B$ to the relative strength of correlation between the types $A, B$. It may be noted that $v_{\mathrm{dyn}}[A, B]$ should be zero if particles $A$ and $B$ are produced in statistically independent way $[32,35,43]$. In practice, however, a nonzero value of $v_{\text {dyn }}[A, B]$ is expected because produced particles are partially correlated through the production of resonances, string fragmentation, jet fragmentation, and (or) other mechanisms [29]. A negative value of $v_{\mathrm{dyn}}[A, B]$ indicates a correlation, whereas positive value would indicate the presence of anticorrelation between particle types $A$ and $B$. The indices $A, B$ are taken as particle pair combinations, such as $[\pi, K],[p, K]$, and $[\pi, p]$ in the present work to construct $v_{\mathrm{dyn}}$.

\section{The HIJING Model}

The Monte Carlo model HIJING (Heavy-Ion Jet Interaction Generator) was developed to study the role of minijets and particle production in proton-proton ( $\mathrm{pp}$ ), proton-nucleus (pA), and nucleus-nucleus (AA) collisions in a wide range of energies from 5 to $2000 \mathrm{GeV}[47,48]$. The HIJING model is commonly used in high-energy heavy-ion collisions for providing the baseline to compare the simulation results with the experimental data. The main feature of the HIJING model is based on pQCD (perturbative QCD) approach considering that the multiple minijet partons produced in collisions are transformed into string fragmentation which, in turn, decays into hadrons. The $\mathrm{pQCD}$ process is 
implemented in HIJING using the PYTHIA $[49,50]$ model for hadronic collisions. The cross section in PQCD for hard parton scattering is determined using leading order to simulate the higher order corrections. The eikonal formalism is embedded to calculate the number of minijets per inelastic nucleon-nucleon collisions. The soft contributions are modeled by diquark-quark strings with gluon kinks along with the line of the Lund FRITIOF and DPM (Dual Parton Model) $[48,51-55]$. Besides this, the basic property of the HIJING model is that it considers the nucleus-nucleus collisions as a superposition of nucleon-nucleon collisions. However, the mechanism for final state interactions among the low $p_{T}$ particles is not included in the HIJING model. Due to which, the phenomena such as collectivity and equilibrium cannot be addressed. Therefore, HIJING is mainly designed to explore the range of possible initial conditions that may occur in high-energy heavy-ion collisions.

Furthermore, HIJING also takes into account other important physics processes like jet quenching [56], multiple scattering, and nuclear shadowing to study the nuclear effects [48]. To study the dependence of moderate and high $p_{T}$ observables on an assumed energy loss of partons traversing the produced dense matter, a jet quenching approach is incorporated in the HIJING model [48].

In high-energy heavy-ion collisions, the interaction of high $p_{T}$ jets in the produced transient dense medium is treated as one of the signals of phase transition [56]. Therefore, the rapid variation of $\mu_{D}$ (Debye screening) near the phase transition point could lead to a variation of jet quenching phenomenon that could be used as a diagnostic tool of the QGP phase transition [48]. Furthermore, resonances play an important role in studying the net charge fluctuations. Resonances have short lifetime and subsequently decay into stable hadrons. This would affect the final hadron yields and their number fluctuations [57]. Resonance decay kinematics influences charge fluctuations in two different ways. It dilutes the effect of global charge conservation if only one of the decay products falls into the acceptance window. However, if both decay products lie within the acceptance cone, mean charged particle multiplicity will increase but the net charge does not change [58]. Hadron production in HIJING involves a cocktail of resonances that may also give a rough estimate of the strength of correlations between charged and neutral kaons [59]. The present study is an attempt to explore the effect of fluctuations in understanding the dissipative properties of a color-defined medium using the jet quenching, resonance production, and jet/minijet contributions incorporated in the HIJING model [60]. It was found that [61-64] the HIJING predicted values of charged particle density when jet quenching and contributions from resonance decays are switched off, which are consistent with the ones observed in $\mathrm{Au}-\mathrm{Au}$ collisions at $200 \mathrm{GeV}$ per nucleon and $\mathrm{Pb}-\mathrm{Pb}$ at 2.76 and $5.5 \mathrm{TeV}$ per nucleon.

\section{Results and Discussion}

MC events corresponding to $\mathrm{Pb}-\mathrm{Pb}$ at $\sqrt{s}_{N N}=2.76$ and 5.02 and $\mathrm{Xe}-\mathrm{Xe}$ collisions at $\sqrt{s}_{N N}=5.44 \mathrm{TeV}$ are generated using the HIJING-1.37 [47, 48]. Events are simulated by running
TABLE 1: Details of events simulated for the analysis.

\begin{tabular}{|c|c|c|c|}
\hline $\begin{array}{l}\text { Energy } \\
(\mathrm{GeV})\end{array}$ & $\begin{array}{l}\text { Type of } \\
\text { collision }\end{array}$ & $\begin{array}{l}\text { Analysis } \\
\text { mode }\end{array}$ & $\begin{array}{l}\text { No. of events } \\
\quad\left(\times 10^{6}\right)\end{array}$ \\
\hline \multirow{3}{*}{2760} & \multirow{3}{*}{$\mathrm{Pb}-\mathrm{Pb}$} & Res-off JQ-on & 4.4 \\
\hline & & Res-off JQ-off & 3.6 \\
\hline & & Res-on JQ-off & 2.3 \\
\hline \multirow{3}{*}{5020} & \multirow{3}{*}{$\mathrm{Pb}-\mathrm{Pb}$} & Res-off JQ-on & 3.3 \\
\hline & & Res-off JQ-off & 2.5 \\
\hline & & Res-on JQ-off & 2.3 \\
\hline \multirow{3}{*}{5440} & \multirow{3}{*}{$\mathrm{Xe}-\mathrm{Xe}$} & Res-off JQ-on & 2.9 \\
\hline & & Res-off JQ-off & 2.5 \\
\hline & & Res-on JQ-off & 3.7 \\
\hline
\end{tabular}

the code in three different modes: (i) HIJING default, i.e., resonance- (Res-) off jet quenching- (JQ-) off, (ii) Res-on JQ-off, and (iii) Res-off JQ-on. The number of events simulated in each of these modes is listed in Table 1 . The analysis is carried out by considering only those charged particles which have pseudorapidity $(\eta)$ and transverse momentum $\left(p_{T}\right)$ in the range $|\eta|<0.8$ and $0.2<p_{T}<1.5 \mathrm{GeV} / \mathrm{c}$, respectively. The ALICE experiment has also used the same $\eta$-cut, but instead of $p_{T}$, they have considered the charged particles with momentum, $0.2<p<1.5 \mathrm{GeV} / \mathrm{c}$. It may be mentioned here that for the $\eta$ range considered in the ALICE experiment and also in the present study, for $p_{T}<5.0 \mathrm{GeV} / \mathrm{c}$, $p_{T} \simeq 0.9 p$. In order to examine the effect of jet quenching, a higher $p_{T}$ range, $0.2<p_{T}<5.0 \mathrm{GeV} / \mathrm{c}$, is also considered where this effect is expected to be more visible. The centrality of an event is estimated by applying VZERO-A and VZERO-C detector $\eta$ cuts of the ALICE experiment [65-67], i.e., by considering the charged particles which have their $\eta$ values in the range $2.8<\eta<5.1$ or $-3.7<\eta<-1.7$. For this multiplicity, distributions of charged particles having their $\eta$ values within these limits are examined and quantiled to fix the minimum and maximum limits for a centrality class.

The values of mean number of participating nucleons $\left\langle N_{\text {part }}\right\rangle$ and mean charged particle density $\left\langle d N_{\mathrm{ch}} / d \eta\right\rangle$ for different centrality classes are listed in Tables $2-4$. Variations of $\left\langle d N_{\mathrm{ch}} / d \eta\right\rangle$ with $\left\langle N_{\text {part }}\right\rangle$ for these events are plotted in Figure 1. The values of $\left\langle N_{\text {part }}\right\rangle$ and $\left\langle d N_{\mathrm{ch}} / d \eta\right\rangle$ reported earlier [65-67] are also given in these tables and displayed in the figure. It is interesting to note from Tables $2-4$ and Figure 1 that HIJING default predicts somewhat higher values of $\left\langle N_{\text {part }}\right\rangle$ and $\left\langle d N_{\text {ch }} / d \eta\right\rangle$ for various centrality classes as compared to those observed in experiments $[65,66]$. It may also be noted from the tables and the figure that the values of $\left\langle d N_{\mathrm{ch}} / d \eta\right\rangle$ are higher when jet quenching is turned on, which might be due to the enhanced production of low $p_{T}$ particles. This may be understood as when a partonic jet is quenched in the dense medium, it would fragment into large number of partons which, in turn, result in the production of low $p_{T}$ charged particles [68]. It may also be noted that the effect of jet quenching is rather more pronounced in central 
TABLE 2: Values of $\left\langle N_{\text {part }}\right\rangle$ and $\left\langle d N_{\mathrm{ch}} / d \eta\right\rangle$, in different centrality bins for three tunes of HIJING: (i) default, (ii) Res-off JQ-on, and (iii) Res-on JQ-off for $\mathrm{Pb}-\mathrm{Pb}$ collisions at $\sqrt{s}_{N N}=2.76 \mathrm{TeV}$. The values within brackets are from the experiments, taken from ref. [65]*

\begin{tabular}{lcccccc}
\hline $\begin{array}{l}\text { Centrality } \\
(\%)\end{array}$ & $\left\langle N_{\text {part }}\right\rangle$ & HIJING Res-off JQ-off & \multicolumn{2}{c}{ HIJING Res-off JQ-on } & \multicolumn{2}{c}{ HIJING Res-on JQ-off } \\
\hline $0-5$ & $384.12 \pm 0.16(382.8 \pm 3.1)$ & $1891.4 \pm 0.64(1601 \pm 60)$ & $383.0 \pm 0.12$ & $3609.66 \pm 3.88$ & $383.48 \pm 0.16$ & $3086.72 \pm 2.57$ \\
$5-10$ & $326.94 \pm 0.15(329.7 \pm 4.6)$ & $1556.96 \pm 0.47(1294 \pm 49)$ & $326.1 \pm 0.14$ & $2885.69 \pm 2.97$ & $325.49 \pm 0.14$ & $2352.9 \pm 1.81$ \\
$10-20$ & $250.03 \pm 0.21(260.5 \pm 4.4)$ & $1198.73 \pm 0.42(966 \pm 37)$ & $249.8 \pm 0.21$ & $2114.36 \pm 2.70$ & $250.29 \pm 0.21$ & $1727.05 \pm 0.97$ \\
$20-30$ & $170.60 \pm 0.18(186.4 \pm 3.9)$ & $841.22 \pm 0.31(649 \pm 23)$ & $170.9 \pm 0.17$ & $1387.39 \pm 1.93$ & $170.64 \pm 0.17$ & $1169.5 \pm 0.61$ \\
$30-40$ & $115.52 \pm 0.14(128.9 \pm 3.3)$ & $576.68 \pm 0.24(426 \pm 15)$ & $111.5 \pm 0.14$ & $870.71 \pm 0.02$ & $111.55 \pm 0.14$ & $775.09 \pm 0.38$ \\
$40-50$ & $68.57 \pm 0.12(85.0 \pm 2.6)$ & $381.47 \pm 0.18(261 \pm 9)$ & $68.9 \pm 0.11$ & $519.95 \pm 0.92$ & $68.32 \pm 0.11$ & $490.03 \pm 0.23$ \\
$50-60$ & $38.87 \pm 0.09(52.8 \pm 2.0)$ & $239.56 \pm 0.13(149 \pm 6)$ & $38.7 \pm 0.08$ & $296.59 \pm 0.60$ & $39.20 \pm 0.08$ & $293.2 \pm 0.13$ \\
$60-70$ & $20.29 \pm 0.06(30.3 \pm 1.3)$ & $141.69 \pm 0.09(76 \pm 4)$ & $20.0 \pm 0.06$ & $157.44 \pm 0.38$ & $20.21 \pm 0.06$ & $165.93 \pm 0.08$ \\
$70-80$ & $10.33 \pm 0.04(15.8 \pm 0.6)$ & $79.14 \pm 0.06(35 \pm 2)$ & $10.5 \pm 0.04$ & $79.57 \pm 0.22$ & $10.44 \pm 0.04$ & $88.10 \pm 0.05$ \\
\hline
\end{tabular}

${ }^{*}$ Errors associated include systematic and statistical errors.

TABLE 3: Values of $\left\langle N_{\text {part }}\right\rangle$ and $\left\langle d N_{\mathrm{ch}} / d \eta\right\rangle$ for $\mathrm{Pb}-\mathrm{Pb}$ collisions at $\sqrt{s}_{N N}=5.02 \mathrm{TeV}$. The same details are mentioned in Table 2. The values within brackets have been obtained from the data as reported in ref. [66]*.

\begin{tabular}{lcccccc}
\hline $\begin{array}{l}\text { Centrality } \\
(\%)\end{array}$ & $\left\langle N_{\text {part }}\right\rangle$ & HIJING Res-off JQ-off & \multicolumn{2}{c}{ HIJING Res-off JQ-on } & \multicolumn{3}{c}{ HIJING Res-on JQ-off } \\
\hline $0-5$ & $385.82 \pm 0.11(385.0 \pm 2.5)$ & $2338.57 \pm 0.5(1943 \pm 54)$ & $385.80 \pm 0.11$ & $4463.09 \pm 1.52$ & $385.88 \pm 0.16$ & $3762.82 \pm 3.74$ \\
$5-10$ & $331.04 \pm 0.14(333.0 \pm 4.0)$ & $1847.71 \pm 0.36(1586 \pm 46)$ & $330.79 \pm 0.14$ & $3344.38 \pm 0.94$ & $330.57 \pm 0.14$ & $2774.66 \pm 2.39$ \\
$10-20$ & $254.51 \pm 0.21(263.0 \pm 4.0)$ & $1344.25 \pm 0.29(1180 \pm 31)$ & $254.04 \pm 0.2$ & $2348.47 \pm 0.78$ & $254.12 \pm 0.21$ & $1942.26 \pm 1.29$ \\
$20-30$ & $174.85 \pm 0.18(188.0 \pm 3.0)$ & $908.39 \pm 0.19(786 \pm 20)$ & $175.31 \pm 0.14$ & $1489.35 \pm 0.50$ & $174.89 \pm 0.17$ & $1266.45 \pm 0.71$ \\
$30-40$ & $116.56 \pm 0.15(131.0 \pm 2.0)$ & $615.52 \pm 0.14(512 \pm 15)$ & $117.15 \pm 0.14$ & $928.62 \pm 0.34$ & $116.18 \pm 0.14$ & $815.25 \pm 0.42$ \\
$40-50$ & $72.26 \pm 0.12(86.3 \pm 1.7)$ & $406.44 \pm 0.10(318 \pm 12)$ & $71.23 \pm 0.11$ & $556.89 \pm 0.23$ & $72.10 \pm 0.11$ & $510.12 \pm 0.24$ \\
$50-60$ & $41.77 \pm 0.09(53.6 \pm 1.2)$ & $257.53 \pm 0.07(183 \pm 8)$ & $41.82 \pm 0.08$ & $320.21 \pm 0.15$ & $41.83 \pm 0.09$ & $306.40 \pm 0.14$ \\
$60-70$ & $22.31 \pm 0.06(30.4 \pm 0.8)$ & $155.53 \pm 0.05(96.3 \pm 5.8)$ & $22.09 \pm 0.06$ & $175.42 \pm 0.09$ & $21.98 \pm 0.06$ & $174.66 \pm 0.08$ \\
$70-80$ & $11.57 \pm 0.04(15.6 \pm 0.5)$ & $88.87 \pm 0.03(44.9 \pm 3.4)$ & $11.48 \pm 0.04$ & $91.58 \pm 0.06$ & $11.47 \pm 0.04$ & $94.18 \pm 0.05$ \\
\hline
\end{tabular}

*Errors associated include systematic and statistical errors.

TABLe 4: Values of $\left\langle N_{\text {part }}\right\rangle$ and $\left\langle d N_{\mathrm{ch}} / d \eta\right\rangle$ for $5.44 \mathrm{TeV}$ Xe-Xe collisions. The other details are as given in Table 2. Experimentally observed values, given within brackets, are taken from ref. [67]*

\begin{tabular}{|c|c|c|c|c|c|c|}
\hline \multirow{2}{*}{$\begin{array}{l}\text { Centrality } \\
(\%)\end{array}$} & \multicolumn{2}{|c|}{ HIJING Res-off JQ-off } & \multicolumn{2}{|c|}{ HIJING Res-off JQ-on } & \multicolumn{2}{|c|}{ HIJING Res-on JQ-off } \\
\hline & $\left\langle N_{\text {part }}\right\rangle$ & $\left\langle d N_{\mathrm{ch}} / d \eta\right\rangle$ & $\left\langle N_{\text {part }}\right\rangle$ & $\left\langle d N_{\mathrm{ch}} / d \eta\right\rangle$ & $\left\langle N_{\text {part }}\right\rangle$ & $\left\langle d N_{\mathrm{ch}} / d \eta\right\rangle$ \\
\hline $0-5$ & $231.04 \pm 0.03(236.0 \pm 2.0)$ & $1518.05 \pm 0.33(1167 \pm 26)$ & $231.31 \pm 0.04$ & $2983.14 \pm 0.76$ & $236.50 \pm 0.03$ & $1787.88 \pm 0.33$ \\
\hline $5-10$ & $202.44 \pm 0.04(207.0 \pm 3.0)$ & $1211.96 \pm 0.25(939 \pm 24)$ & $201.53 \pm 0.04$ & $2288.45 \pm 0.54$ & $208.43 \pm 0.03$ & $1447.17 \pm 0.26$ \\
\hline $10-20$ & $160.91 \pm 0.04(165.0 \pm 3.0)$ & $891.22 \pm 0.22(706 \pm 17)$ & $159.98 \pm 0.04$ & $1591.45 \pm 0.47$ & $165.96 \pm 0.03$ & $1059.88 \pm 0.23$ \\
\hline $20-30$ & $114.89 \pm 0.03(118.0 \pm 4.0)$ & $579.72 \pm 0.15$ & $114.26 \pm 0.03$ & $956.1 \pm 0.31$ & $119.15 \pm 0.03$ & \pm 0.16 \\
\hline $30-40$ & $79.50 \pm 0.02(82.2 \pm 3.9)$ & $363.21 \pm 0.11(315 \pm 8)$ & $79.0 \pm 0.02$ & $542.51 \pm 0.21$ & $82.79 \pm 0.02$ & $430.86 \pm 0.11$ \\
\hline $40-50$ & $52.69 \pm 0.02(54.6 \pm 3.6)$ & $214.87 \pm 0.08(198 \pm 5)$ & $52.29 \pm 0.02$ & $287.15 \pm 0.13$ & $55.15 \pm 0.02$ & $255.61 \pm 0.08$ \\
\hline $50-60$ & $32.92 \pm 0.01(34.1 \pm 3.0)$ & $118.64 \pm 0.05(118 \pm 3$ & $32.76 \pm 0.01$ & $142.38 \pm 0.07$ & $34.77 \pm 0.01$ & $141.99 \pm 0.05$ \\
\hline $60-70$ & $19.28 \pm 0.01(19.7 \pm 2.1)$ & $60.98 \pm 0.03(64.7 \pm 2.0)$ & $19.2 \pm 0.01$ & $66.75 \pm 0.04$ & $20.47 \pm 0.01$ & $73.25 \pm 0.03$ \\
\hline $70-80$ & $10.54 \pm 0.01(10.5 \pm 1.1)$ & $28.88 \pm 0.02(32.0 \pm 1.3)$ & $10.51 \pm 0.01$ & $29.74 \pm 0.02$ & $11.23 \pm 0.01$ & $34.82 \pm 0.02$ \\
\hline
\end{tabular}

*Errors associated include systematic and statistical errors. 

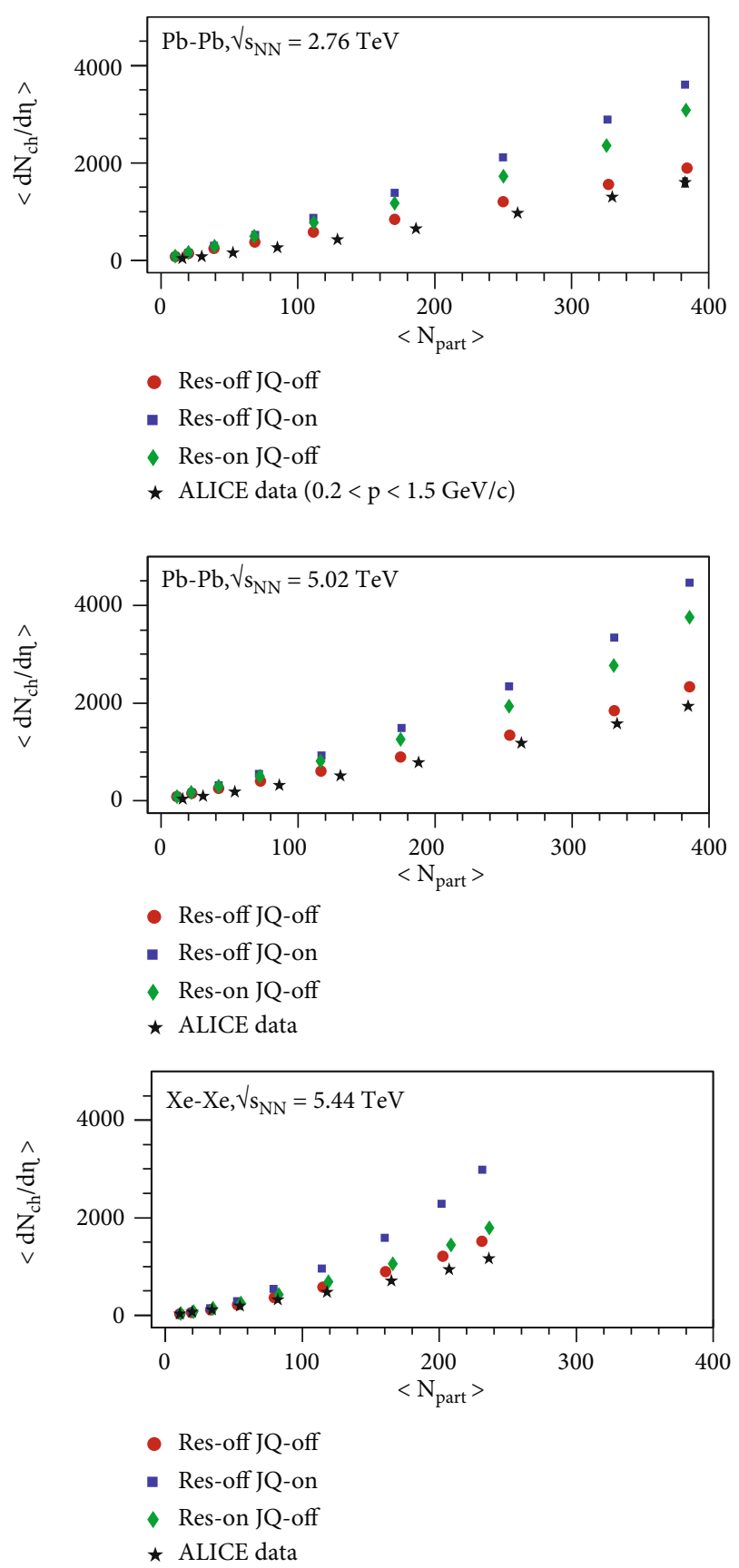

FIgURE 1: Dependence of charged particle density on mean number of participating nucleons for $\mathrm{Pb}-\mathrm{Pb}$ collisions at $\sqrt{s}_{N N}=2.76$ and $5.02 \mathrm{TeV}$ and $\mathrm{Xe}-\mathrm{Xe}$ collisions at $\sqrt{s}_{N N}=5.44 \mathrm{TeV}$. The values reported by ALICE collaboration are taken from refs. [65-67].

collisions, as compared to that in peripheral collisions. Enhancement in the $\left\langle d N_{\mathrm{ch}} / d \eta\right\rangle$ values due to resonances may also be seen in the figure.

Variations of mean multiplicities of charged pions, kaons, protons, and antiprotons with $\left\langle N_{\text {part }}\right\rangle$ are shown in Figure 2. It is observed that mean multiplicities of $\pi^{ \pm}, K^{ \pm}$, and $p \bar{p}$ increase with increasing $\left\langle N_{\text {part }}\right\rangle$ in almost identical fashion. It is also noted that the contributions to the particle multiplicities due to the jet quenching and resonance decays are maximum in most central collisions, which gradually decrease with $\left\langle N_{\text {part }}\right\rangle$ and tend to vanish for $\left\langle N_{\text {part }}\right\rangle$ values corresponding to centrality $\sim 50 \%$ and above. The reasons for the enhancement in particle multiplicities have been discussed in the previous section.

The values of $v_{\text {dyn }}$ for the combinations of particles $[\pi, K],[p, K]$, and $[\pi, p]$ are calculated for various centrality classes using Equation (1). Variations of $v_{\text {dyn }}$ for these species of particles for $0-5 \%$ central collisions with beam energy are shown in Figure 3. Values of $v_{\text {dyn }}$ for these combinations of particles, reported by NA49 [40], STAR [41], and ALICE experiment [43], are also presented in the figure. Kinematical ranges used in these experiments are mentioned in the figure.

The statistical errors associated to $v_{\text {dyn }}$ are too small to be visible in the figure. These errors are determined using the subsample method [40]. The data set is divided into 30 subsamples, and the values $v_{\mathrm{dyn}}[A, B]_{i}$ are calculated for each subsample independently. Using these values of $v_{\text {dyn }}$, the mean and dispersion are estimated as

$$
\begin{gathered}
<v_{\text {dyn }}[A, B]>=\frac{1}{n} \sum v_{\text {dyn }}[A, B]_{i}, \\
\sigma_{v_{\text {dyn }}}=\sqrt{\frac{\sum\left(v_{\text {dyn }}[A, B]_{i}-<v_{\text {dyn }}[A, B]>\right)^{2}}{n-1}} .
\end{gathered}
$$

The statistical error associated is then calculated as

$$
(\text { Error })_{\text {stat }}=\frac{\sigma_{v_{\mathrm{dyn}}}}{\sqrt{n}}
$$

The following observations may be made from the figure:

(i) $v_{\text {dyn }}[\pi, K]$ measured by STAR and ALICE experiments [41, 43] acquire positive and nearly energy-independent values from $\sqrt{s}_{N N}=7.7 \mathrm{GeV}$ to $2.76 \mathrm{TeV}$. The HIJING estimated values for $\sqrt{s}_{N N}=2.76 \mathrm{TeV} \mathrm{Pb}-\mathrm{Pb}$ collisions in the present study are observed to be close to those reported by the ALICE experiment. The values of $v_{\text {dyn }}[\pi, K]$ for $0-3.5 \% \mathrm{~Pb}-\mathrm{Pb}$ collisions also match with the STAR findings at $\sqrt{s}_{N N}=11.5$ and $19.6 \mathrm{GeV}$ while below $\sqrt{s}_{N N}=11.5 \mathrm{GeV}$ an increasing trend in $\nu_{\text {dyn }}[\pi, K]$ is seen with decreasing beam energy. Such a difference in $v_{\text {dyn }}[\pi, K]$ values observed in NA49 [40] and STAR [41] experiments has been argued to be due to the difference in measurement methods adopted in the two experiments. The observed positive values of $v_{\mathrm{dyn}}[\pi, K]$ in experiments from $\sqrt{s_{N N}}=7.7 \mathrm{GeV}$ to $2.76 \mathrm{TeV}$ as well as predicted by UrQMD, HSD [41], and HIJING in the present study are either due to the dominance of variance of $K$ and $\pi$ or because of the presence 

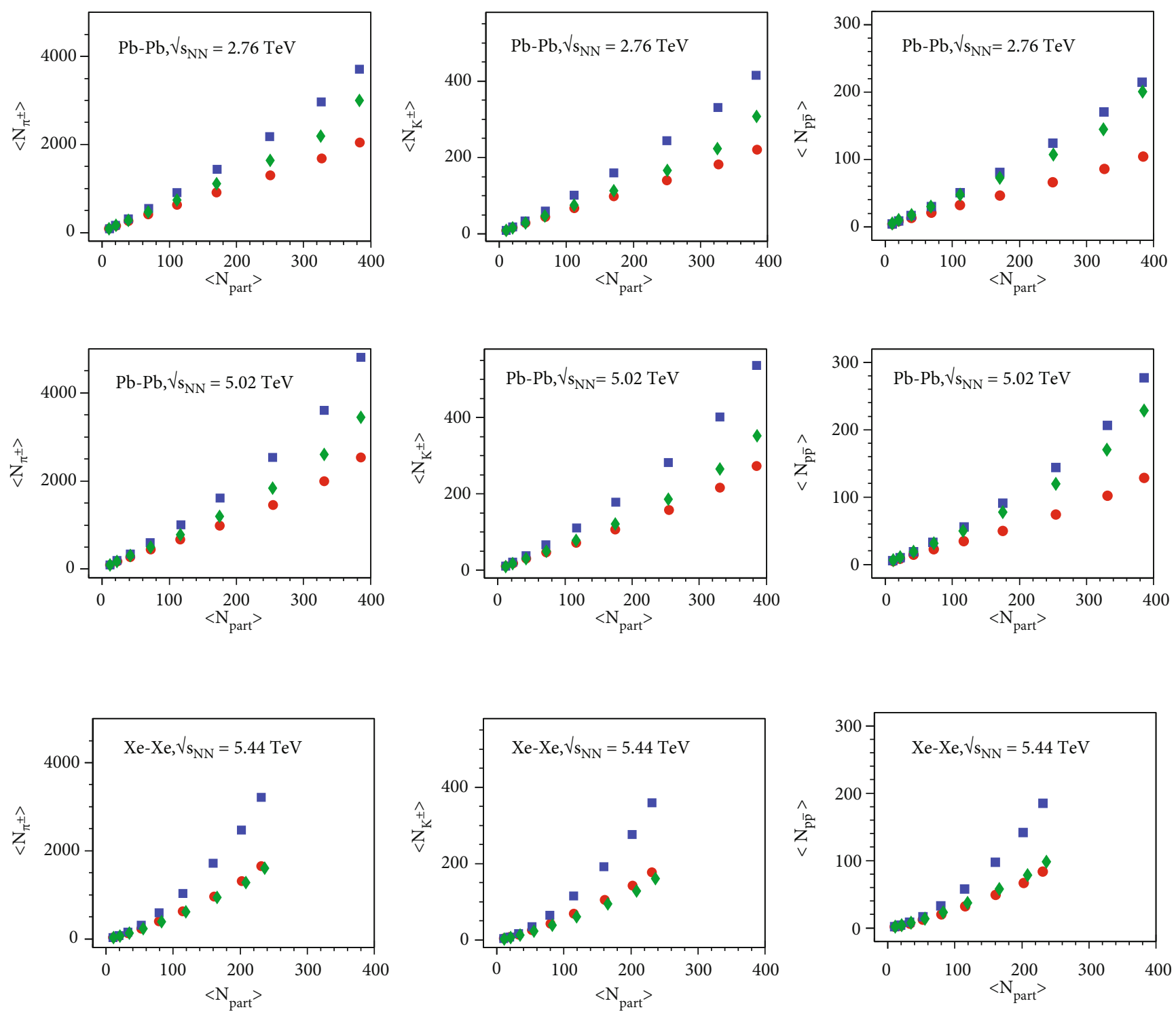

- Res-off JQ-off

- Res-off JQ-on

Res-on JQ-off

Figure 2: Variations of $\left\langle N_{\mathrm{ch}}\right\rangle$ with $\left\langle N_{\text {part }}\right\rangle$ for $\pi^{ \pm}, K^{ \pm}$, and $p \bar{p}$ in $\mathrm{Pb}-\mathrm{Pb}$ and $\mathrm{Xe}-\mathrm{Xe}$ collisions at $\sqrt{s}_{N N}=2.76,5.02$, and 5.44 TeV. The transverse momentum range is $0.2<p_{T}<1.5 \mathrm{GeV} / \mathrm{c}$.

of an anticorrelation $\left(\left\langle N_{\pi} N_{K}\right\rangle<0\right)$ between $K$ and $\pi$

(ii) $v_{\text {dyn }}[p, K]$ values, as reported by STAR [41] and ALICE [43], may be observed to show an increasing trend with beam energy. At $\sqrt{s}_{N N}=7.7 \mathrm{GeV}$, the value is maximum negative, approaches to zero at $\sqrt{s}_{N N}=200 \mathrm{GeV}$, and becomes positive for $\mathrm{Pb}-\mathrm{Pb}$ collisions at $\sqrt{s}_{N N}=2.76 \mathrm{TeV}$. This indicates that the correlation between kaons and protons decreases with increasing incident energy. The HIJING values observed at $\sqrt{s}_{N N}=2.76 \mathrm{TeV}$ are close to the experimental results. The HIJING data points for $\sqrt{s}_{N N}=5.02 \mathrm{TeV} \mathrm{Pb}-\mathrm{Pb}$ collisions and $\sqrt{s}_{N N}=5.44 \mathrm{TeV}$ Xe-Xe collisions tend to follow the trend shown by the data, if extrapolated up to these energies. The higher and positive values of $v_{\text {dyn }}[p, K]$ observed by NA49 experiment [40] for $0-3.5 \%$ central $\mathrm{Pb}-\mathrm{Pb}$ collisions might be due to different detector acceptance of NA49 and STAR experiments; detection of particle pairs resulting from the resonance decays is affected by the limited detector acceptance [41]. Studies involving secondorder off-diagonal cumulants in the energy range 

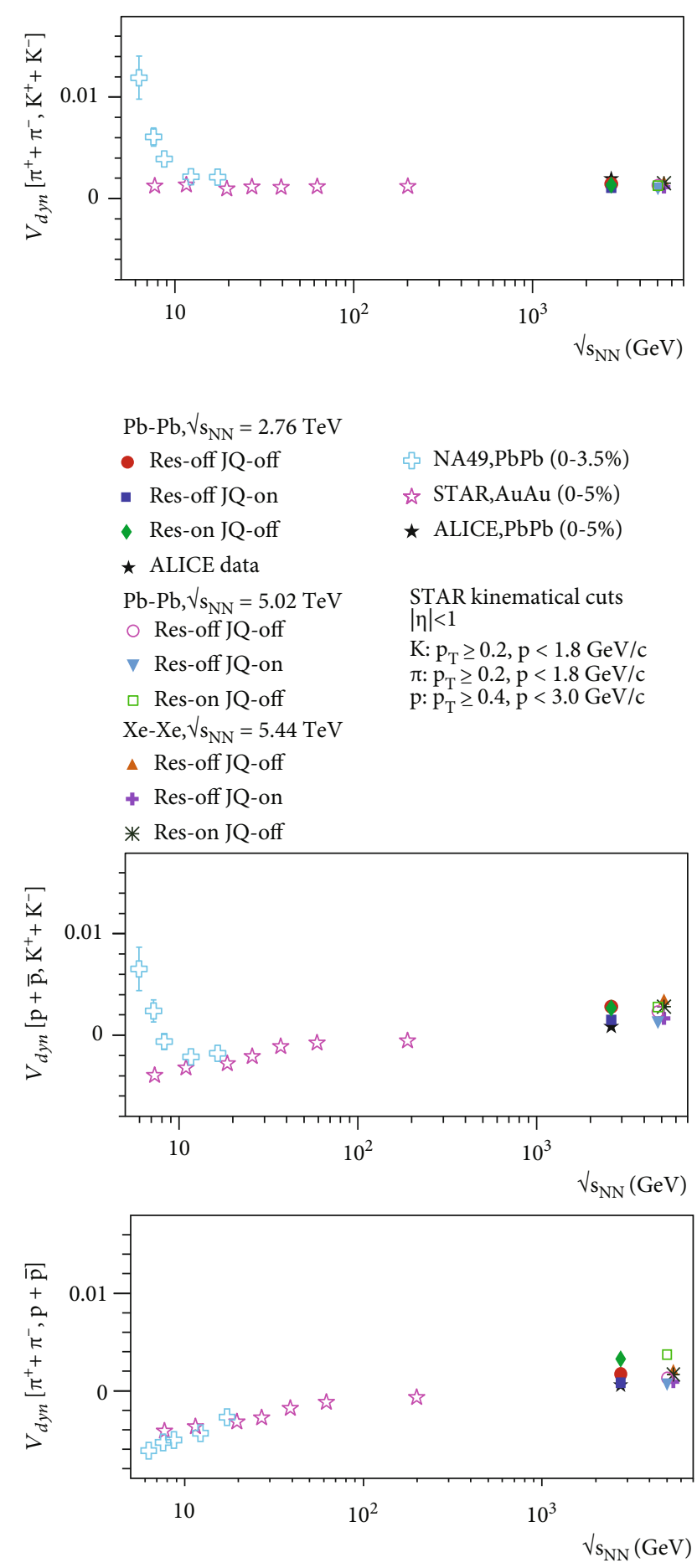

FIGURE 3: Energy dependence of $v_{\text {dyn }}$ for $[\pi, K],[p, K]$, and $[\pi, p]$ for HIJING default, Res-off JQ-on, and Res-on JQ-off for 0-5\% central events. Values corresponding to STAR, NA49, and ALICE experiments are taken from refs. [40, 41, 43].

$\sqrt{s}_{N N}=7.7$ to $200 \mathrm{GeV}$ carried out by STAR experiment [69] show that the correlations between net proton and net kaon multiplicity distributions are negative at $\sqrt{s}_{N N}=200 \mathrm{GeV}$. It increases with decreasing beam energy, changes sign at $\sqrt{s}_{N N}=$ $19.6 \mathrm{GeV}$, and is maximum at $\sqrt{s}_{N N}=7.7 \mathrm{GeV}$. The possible reason for the positive correlation between net proton and net kaons might be due to the associated production: $p p \longrightarrow p \Lambda(1115) K^{+}$ [70], which would give events having higher net proton to be associated to higher net kaons at lower energies. It has been argued that the negative correlation between $p$ and $k$ is expected to arise from QGP phase, where $T-\mu_{B}$ dependence is weak. Although the model calculations based on nonthermal (UrQMD) and thermal (HRG) production of hadrons do not agree with the experimental results, it is expected that such a data-model comparison using the data with improved tracking capabilities and enhanced acceptance will help to understand the baryon-strangeness correlations which is predicted to have different $T-\mu_{B}$ dependence in hadronic and QGP phases

(iii) $v_{\text {dyn }}[\pi, p]$ values from STAR [41] and ALICE [43] experiments exhibit almost the similar trend of energy dependence as that in the case of $v_{\text {dyn }}[p, K]$. $\nu_{\text {dyn }}[\pi, p]$ values for $\mathrm{Pb}-\mathrm{Pb}$ collisions at $\sqrt{s}_{N N}=$ $2.76 \mathrm{TeV}$, observed from the HIJING model, when resonance and jet quenching are switched off, are close to the reported experimental values. It may also be noted that the $v_{\mathrm{dyn}}[\pi, p]$ values obtained with resonance turned as "on" are somewhat higher for $\mathrm{Pb}-\mathrm{Pb}$ collisions at both $\sqrt{s}_{N N}=2.76$ and $5.02 \mathrm{TeV}$. This might be because of relative dominance of $\Delta$ resonance production predicted by the model which gives rise to pion-proton correlations as compared to uncorrelated $p \bar{p}$ pair production [41]. The reduction in $\nu_{\text {dyn }}[\pi, p]$ values observed in experiments has been argued to be due to increasing rate of pair production as compared to the rate of $\Delta$ resonance production with increasing beam energy

Variation of $v_{\text {dyn }}$ with $\left\langle N_{\text {part }}\right\rangle$ for the three combinations of particle species is shown in Figure 4 . It is observed that $v_{\text {dyn }}$ is maximum for the smallest value of $\left\langle N_{\text {part }}\right\rangle$, i.e., for peripheral collisions. It decreases quickly as $\left\langle N_{\text {part }}\right\rangle$ becomes larger and thereafter acquires nearly constant positive values for $\left\langle N_{\text {part }}\right\rangle \geq 100$. In order to examine the effect of jet quenching, a parallel analysis of the data considering the $p_{T}$ range $0.2<p_{T}<5.0 \mathrm{GeV} / \mathrm{c}$ is also carried out, because this effect is expected to be more visible on higher $p_{T}$ range. The values of $v_{\text {dyn }}$ for this $p_{T}$ range are plotted against $\left\langle N_{\text {part }}\right\rangle$ in Figure 5 . The values of $v_{\text {dyn }}[A, B]$ for $\mathrm{Xe}-\mathrm{Xe}$ collisions at $\sqrt{s}_{N N}=5.44 \mathrm{TeV}$ are noticed to be larger as compared to those observed for $\mathrm{Pb}-\mathrm{Pb}$ collisions at $\sqrt{s}_{N N}=2.76$ and $5.02 \mathrm{TeV}$ in the region of low $\left\langle N_{\text {part }}\right\rangle$. This indicates the presence of rather stronger anticorrelation in peripheral collisions in the case of smaller systems. The effect of jet quenching and resonance decays also seems to be absent except for very peripheral 

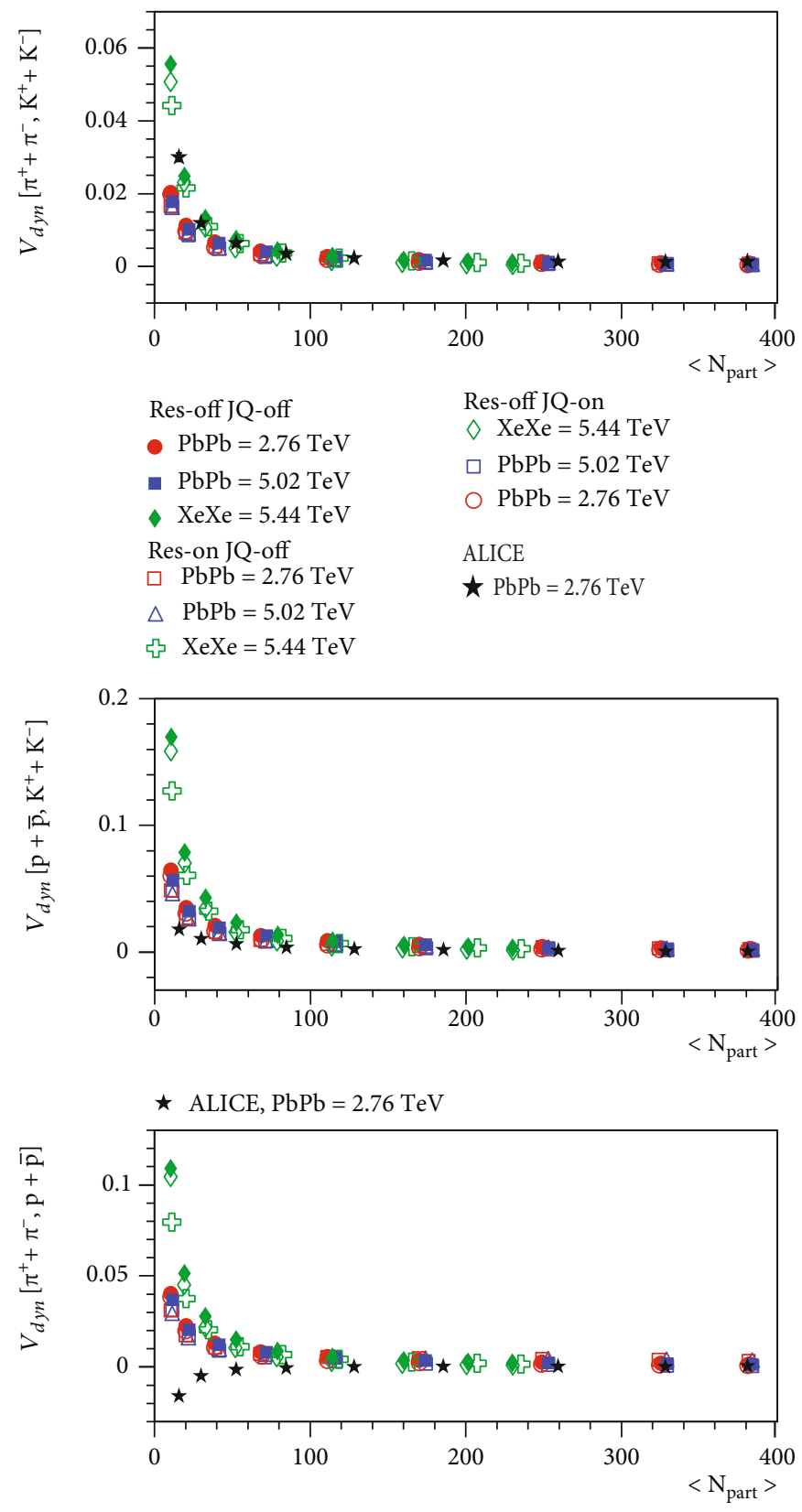

Figure 4: $\left\langle N_{\text {part }}\right\rangle$ dependence of $v_{\text {dyn }}[\pi, k], v_{\text {dyn }}[p, K]$, and $v_{\text {dyn }}[\pi, p]$ for $0.2<p_{T}<1.5 \mathrm{GeV} / c$. Experimental values for $\mathrm{Pb}-\mathrm{Pb}$ collisions at $\sqrt{s}_{N N}=2.76 \mathrm{TeV}$, shown in the figure, are taken from ref. [43].

collisions. For $[\pi, K]$ combination, HIJING predicts slightly smaller values of $v_{\mathrm{dyn}}$ for semicentral and peripheral collisions while for $[p, K]$ pair the model overestimates $v_{\text {dyn }}$ as compared to those obtained from the data [43]. For $[\pi, p]$ combinations, experimental results for $\mathrm{Pb}-\mathrm{Pb}$ collisions at $\sqrt{s}_{N N}=2.76 \mathrm{TeV}$ show that the values of $v_{\text {dyn }}$ decrease with increasing centrality and became more negative for collision centrality $>40 \%$. It may also be noted from Figures 4 and 5 that $v_{\text {dyn }}$ values for various combinations of particle pairs are similar to those obtained with $p_{T}$ cut, $0.2<p_{T}<1.5 \mathrm{GeV} / \mathrm{c}$.
Although $v_{\text {dyn }}$ is robust against detector efficiency losses, yet it has some intrinsic multiplicity dependence $[43,71]$. In order to reduce the effect of multiplicities, the $v_{\text {dyn }}$ values for all three combinations of particles are scaled by mean charged particle density, $\left\langle d N_{\mathrm{ch}} / d \eta\right\rangle$. This removes the $1 / N_{\mathrm{ch}}$ dependence of $v_{\text {dyn }}[32,42]$. The scaled values of $v_{\text {dyn }}[A, B]$ with $\left\langle d N_{\mathrm{ch}} / d \eta\right\rangle$ are plotted as a function of $\left\langle N_{\text {part }}\right\rangle$ for $|\eta|<0.8$ and $p_{T}=0.2$ to 1.5 and 0.2 to $5.0 \mathrm{GeV} / \mathrm{c}$ in Figures 6 and 7 . Experimental results for $\mathrm{Pb}-\mathrm{Pb}$ collisions at $\sqrt{s}_{N N}=2.76$ TeV for $|\eta|<0.8$ and $p=0.2$ to $1.5 \mathrm{GeV} / \mathrm{c}$ are also shown in Figure 6. It may be observed from Figures 6 and 7 that 

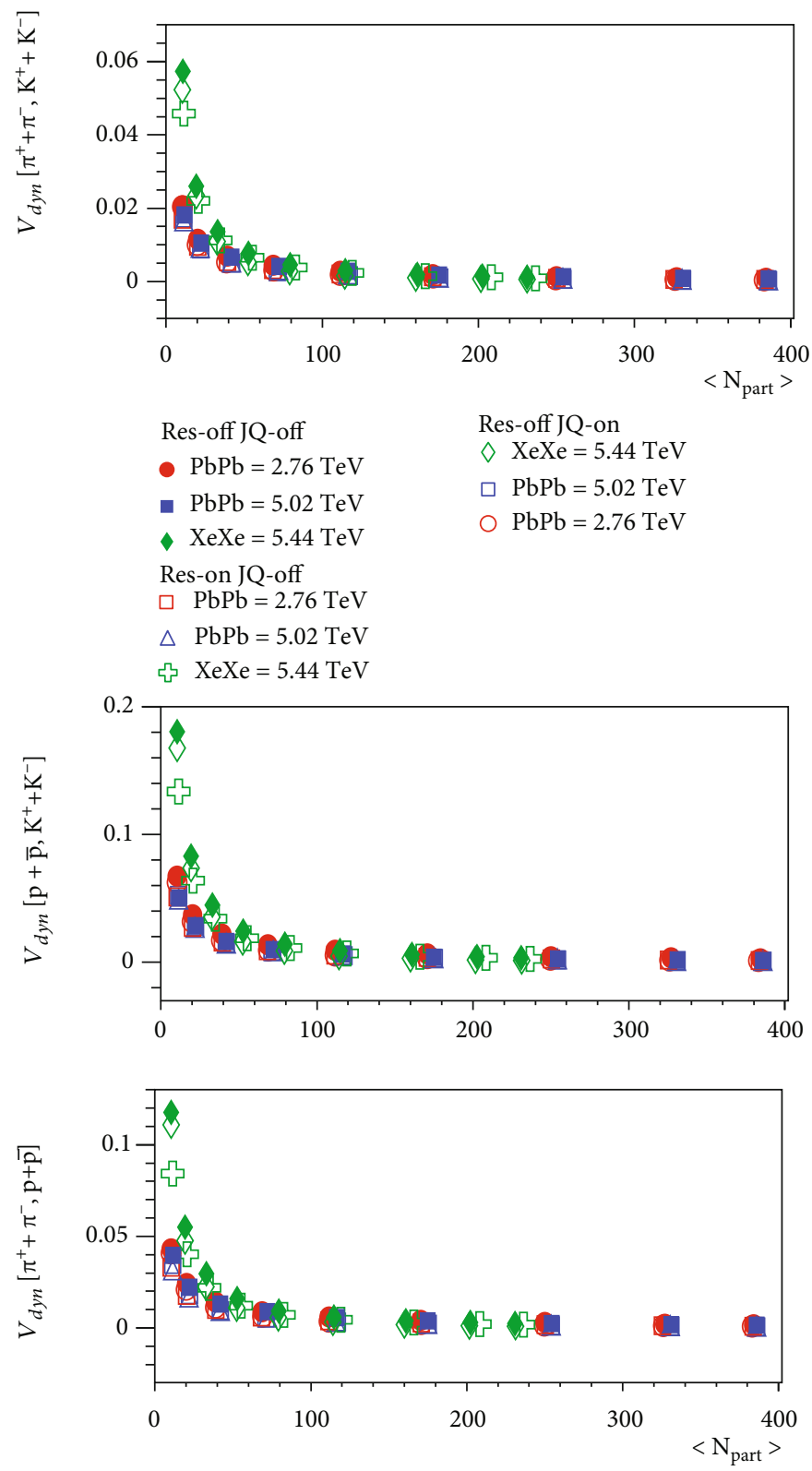

Figure 5: Variations of $v_{\text {dyn }}[\pi, k], v_{\text {dyn }}[p, K]$, and $v_{\text {dyn }}[\pi, p]$ with $\left\langle N_{\text {part }}\right\rangle$ for $0.2<p_{T}<5.0 \mathrm{GeV} / \mathrm{c}$.

(1) $v_{\text {dyn }}[\pi, K]$ scaled values are positive and nearly independent of collision centrality and $p_{T}$ cuts applied. These values are close to those reported by the ALICE experiment [43] for the same $\eta$ cut and $p=0.2$ to $1.5 \mathrm{GeV} / \mathrm{c}$. The effect of jet quenching and resonance decay is, however, noticed to be absent

(2) In case of HIJING default, scaled values of $v_{\text {dyn }}[p, K]$ and $v_{\text {dyn }}[\pi, p]$ for $p_{T}=0.2$ to $1.5 \mathrm{GeV} / \mathrm{c}$ are observed to acquire nearly constant values against centrality and beam energy. These values are however larger than those observed in the ALICE experiment. It is also seen in the figure that the values are higher for central collisions when resonance production is switched on, but on increasing the $p_{T}$ range, i.e., 0.2 to $5.0 \mathrm{GeV} / \mathrm{c}$, the effect of resonance vanishes

The scaled $v_{\text {dyn }}$ values obtained by ALICE collaboration using the HIJING model may be noticed to be positive nearly constant against $d N_{\mathrm{ch}} / d \eta[43,44]$ for all centrality classes and for all the three combinations of particle species. These findings are found to be in good agreement with the values obtained in the present study. This suggests that although HIJING implements global conservation laws yet it does not exhibit any nonmonotonic behaviour as a function of centrality. A comparison of the experimental findings with the AMPT model 

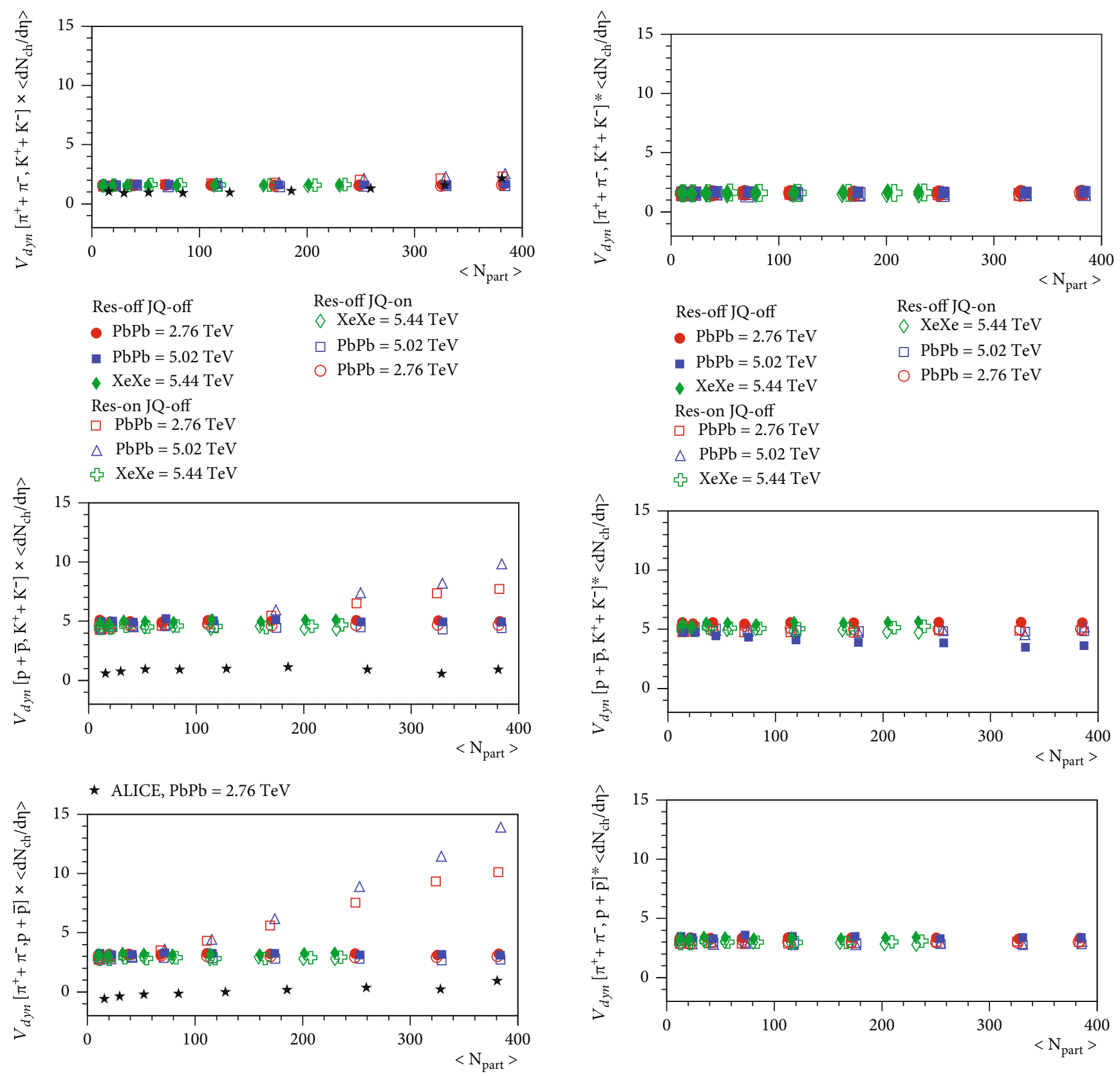

$$
\begin{aligned}
& \text { Res-off JQ-off } \\
& \mathrm{PbPb}=2.76 \mathrm{TeV} \\
& \mathrm{PbPb}=5.02 \mathrm{TeV} \\
& \mathrm{XeXe}=5.44 \mathrm{TeV} \\
& \text { Res-on JQ-off } \\
& \square \mathrm{PbPb}=2.76 \mathrm{TeV} \\
& \triangle \mathrm{PbPb}=5.02 \mathrm{TeV} \\
& \text { ㅈXe }=5.44 \mathrm{TeV}
\end{aligned}
$$
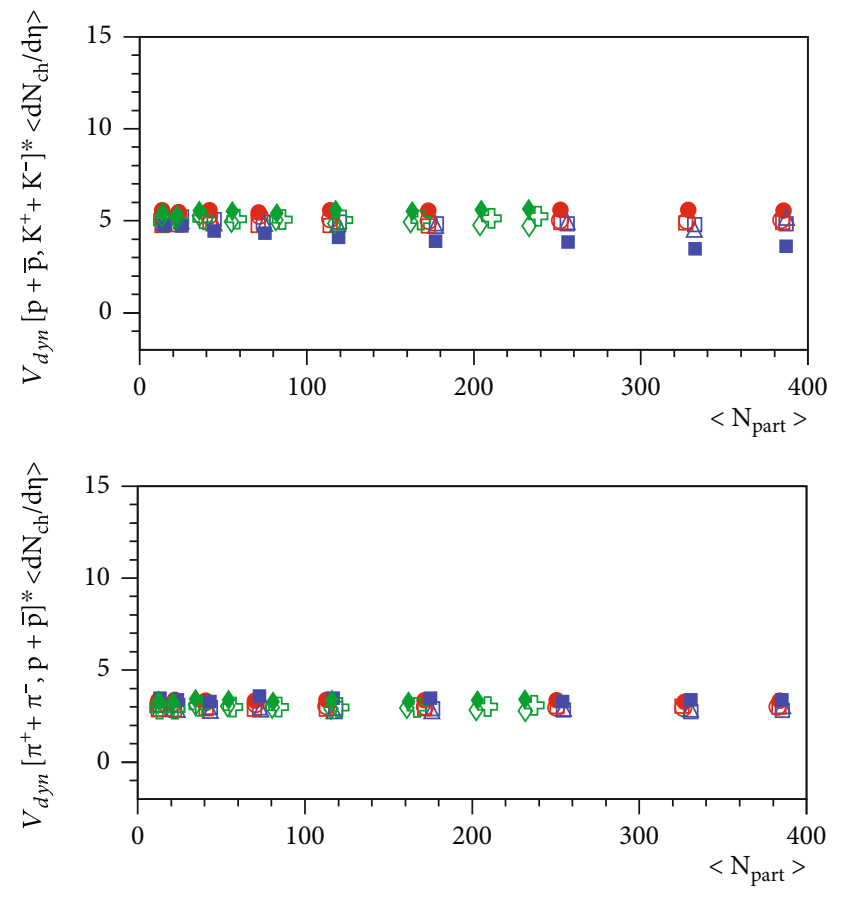

Figure 6: $v_{\text {dyn }}$ scaled by $\left\langle d N_{\text {ch }} / d \eta\right\rangle$ vs. $\left\langle N_{\text {part }}\right\rangle$ for HIJING events in $p_{T}$ range $0.2<p_{T}<1.5 \mathrm{GeV} / \mathrm{c}$. ALICE experimental values, shown in the figure, are taken from ref. [43].

presented in refs. [43] [44] for $2.76 \mathrm{TeV} \mathrm{Pb}-\mathrm{Pb}$ collisions also suggests that AMPT too does not give a quantitative description of the data. However, in AMPT, the resonance production at the hadronization phase due to hadronic rescattering introduces additional correlation between particles, which, in turn, drives $v_{\text {dyn }}$ results toward negative values as the collision centrality increases; particularly for $[\pi, p]$ combination, the AMPT, contrary to the data, predicts negative values for semicentral and central collisions.

FIgURE 7: $v_{\text {dyn }}$ scaled by $\left\langle d N_{\text {ch }} / d \eta\right\rangle$ vs. $\left\langle N_{\text {part }}\right\rangle$ in the $p_{T}$ range 0.2 $<p_{T}<5.0 \mathrm{GeV} / \mathrm{c}$.

Shown in Figure 8 are dependence of $v_{\text {dyn }}[A, B]$ on the mean charged particle density for the three particle type pairs and three tunes of HIJING at the three incident energies. These plots are obtained for the $p_{T}$ range $0.2<p_{T}<$ $1.5 \mathrm{GeV} / \mathrm{c}$. Similar plots for $0.2<p_{T}<5.0 \mathrm{GeV} / \mathrm{c}$ are presented in Figure 9. In order to examine the system size dependence, values of $v_{\text {dyn }}[A, B]$ for $p p$ collision events, simulated using PYTHIA8 [72] at $\sqrt{s}=2.76$ and $5.02 \mathrm{TeV}$ $\left(100 \times 10^{6}\right.$ events in each sample), are also shown in the figures. It is interesting to note from the figures that with increasing $\left\langle d N_{\mathrm{ch}} / d \eta\right\rangle$, the $v_{\mathrm{dyn}}[A, B]$ values decrease first 

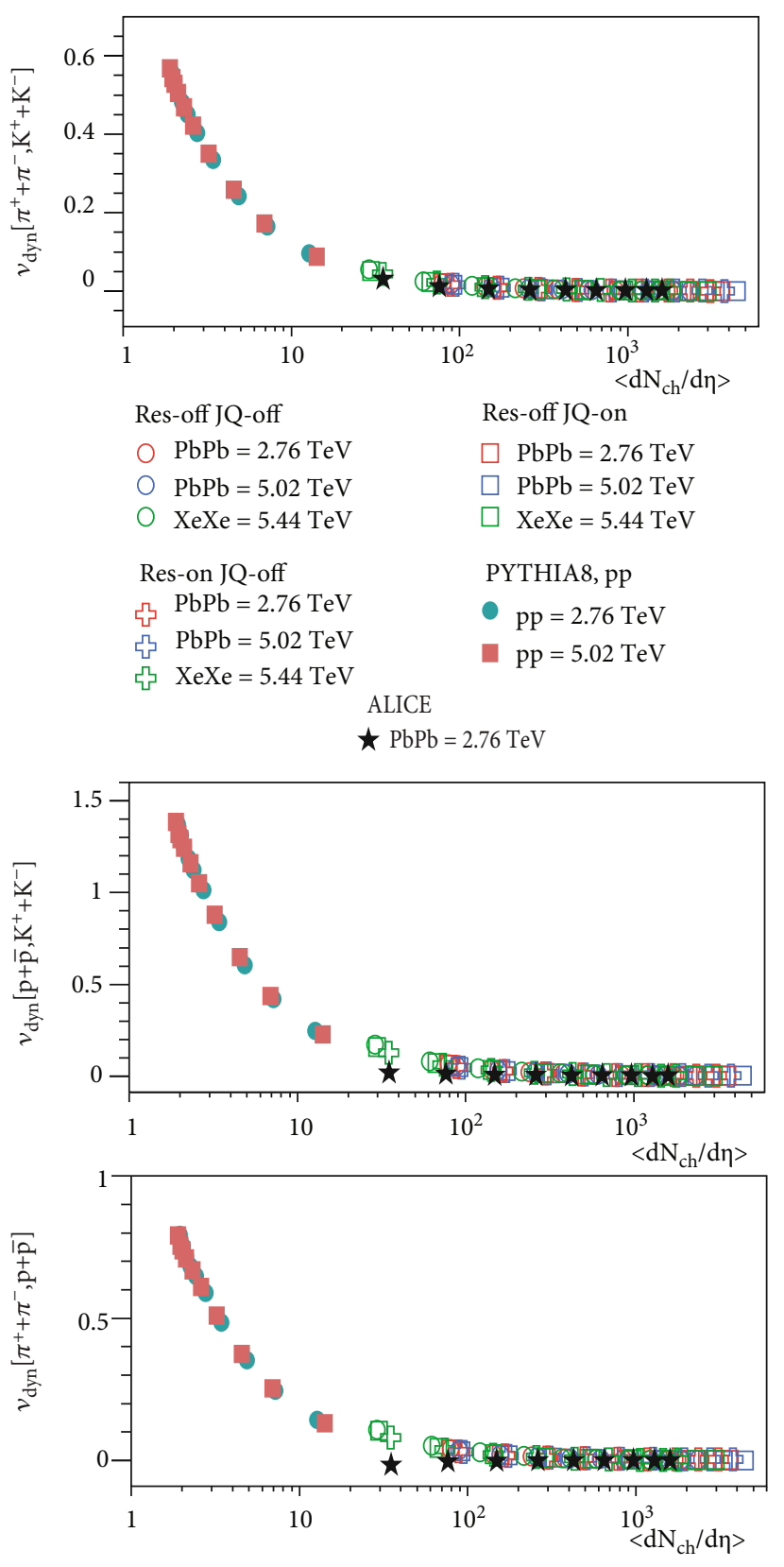

Figure 8: $\left\langle d N_{\mathrm{ch}} / d \eta\right\rangle$ dependence of $v_{\mathrm{dyn}}[\pi, k], v_{\text {dyn }}[p, K]$, and $v_{\text {dyn }}$ $[\pi, p]$ for $p p$ and $\mathrm{Pb}-\mathrm{Pb}$ collisions at $\sqrt{s}_{N N}=2.76$ and $5.02 \mathrm{TeV}$ and Xe-Xe collisions at $\sqrt{s}_{N N}=5.44 \mathrm{TeV}$ in the $p_{T}$ range $0.2<p_{T}$ $<1.5 \mathrm{GeV} / \mathrm{c}$. Experimental results for $\mathrm{Pb}-\mathrm{Pb}$ collisions at $\sqrt{s}_{N N}=$ $2.76 \mathrm{TeV}$, shown in the figure, are taken from ref. [43].

quickly and then slowly and finally tend to saturate for $\left\langle d N_{\mathrm{ch}} / d \eta\right\rangle \sim 100$ and beyond. It may be of interest to note that data points corresponding to the ALICE experiment results [43] overlap the HIJING data for each combination of particle pairs, except for very peripheral collisions where the experimental values for $[p, K]$ and $[\pi, p]$ are noticed to be somewhat smaller. A comparison of the results shown in Figures 8 and 9 indicates that $v_{\text {dyn }}$ values, if plotted against $\left\langle d N_{\mathrm{ch}} / d \eta\right\rangle$, essentially exhibit similar trends irrespective of the $p_{T}$ range considered.
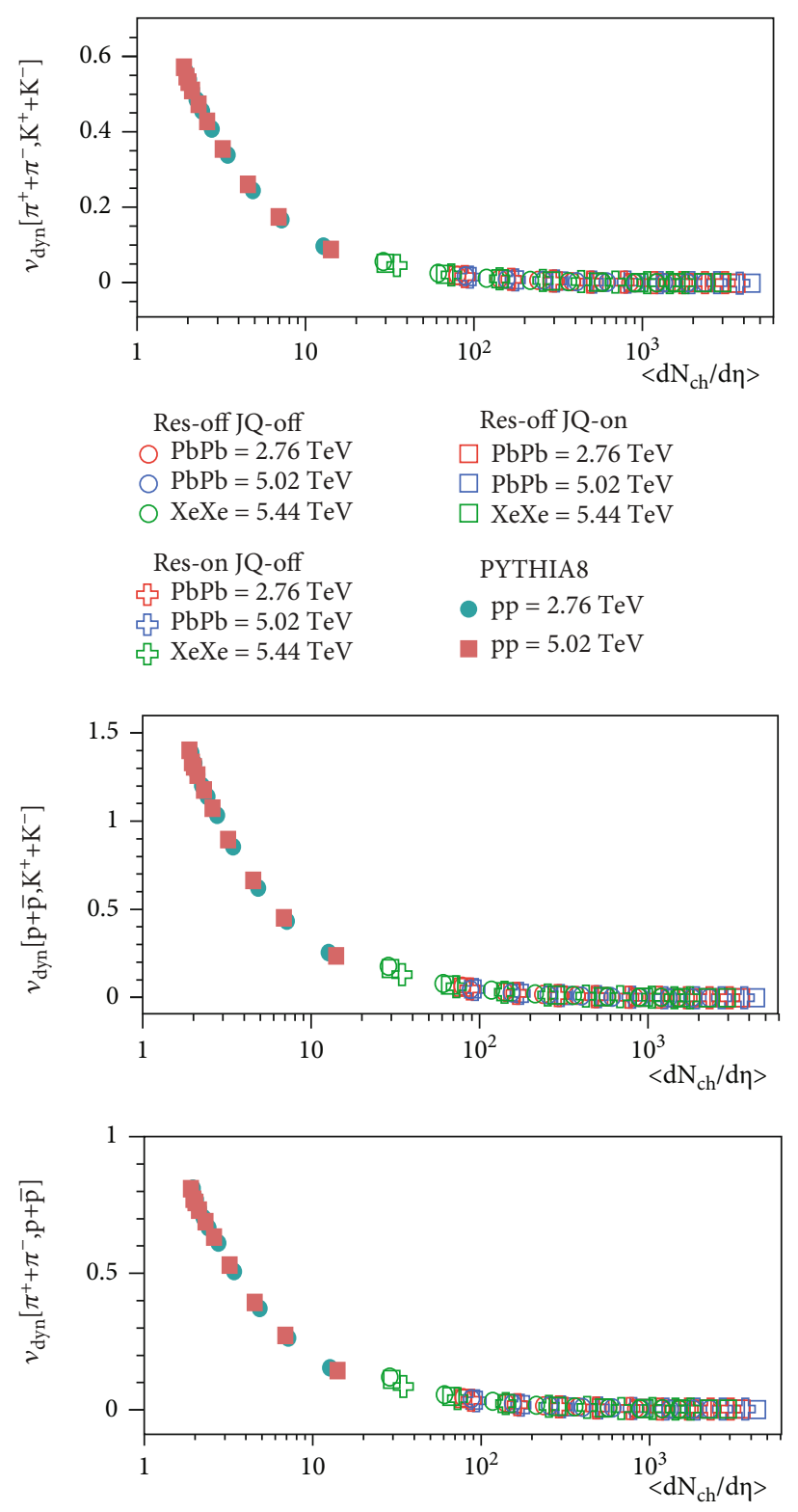

FIgURE 9: $\left\langle d N_{\mathrm{ch}} / d \eta\right\rangle$ dependence of $v_{\text {dyn }}[\pi, k], v_{\text {dyn }}[p, K]$, and $v_{\text {dyn }}$ $[\pi, p]$ for various collision systems at $\sqrt{s}_{N N}=2.76,5.02$, and $5.44 \mathrm{TeV}$ in the $p_{T}$ range $0.2<p_{T}<5.0 \mathrm{GeV} / \mathrm{c}$.

\section{Conclusions}

On the basis of the findings of the present work, the following conclusions may be arrived at:

(1) For most central collisions (0-5\%), HIJING predicted values of $v_{\text {dyn }}$ for the three particle pairs follow the same trend as exhibited by the experimental data from STAR and ALICE experiments

(2) Values of $v_{\text {dyn }}$ for the three combinations of particle pairs for semicentral and peripheral collisions for $\mathrm{Xe}$-Xe collisions are larger than those obtained for $\mathrm{Pb}-\mathrm{Pb}$ collisions at $\sqrt{s}_{N N}=2.76$ and $5.02 \mathrm{TeV}$. This 
difference becomes larger with increasing collision centrality

(3) A comparison of these findings with those reported by the ALICE experiment for $\mathrm{Pb}-\mathrm{Pb}$ collisions at $\sqrt{s}_{N N}=2.76 \mathrm{TeV}$ indicates that for $[\pi, K]$ pair HIJING underestimates, while for $[p, K]$ and $[\pi, p]$ pairs, the model overestimates the $v_{\text {dyn }}$ values. The observed difference in the HIJING predicted and experimental values increases on moving from central to peripheral collisions. The observed lower values of $\nu_{\text {dyn }}[\pi, K]$ against centrality as compared to the experimental results suggest that there might be an anticorrelation between $\pi$ and $K$ in the model or/and the multiplicity distributions of $K$ and $\pi$ are broader. The higher values of $v_{\text {dyn }}$ for $[p, K]$ and $[\pi, p]$ combinations predicted by HIJING, as compared to the experimental findings, may be due to rather weaker $k-p$ and $\pi-p$ correlations

(4) $v_{\text {dyn }}$ values for $p_{T}$ range 0.2 to $5.0 \mathrm{GeV} / c$, when scaled with charged particle density, are observed to acquire nearly constant values against collision centrality and beam energy. The effect of jet quenching and resonances is also observed to be absent. However, for a lower $p_{T}$ range, a significant contribution from the resonance decays in the case of $\mathrm{Pb}-\mathrm{Pb}$ collisions is observed for $[\pi, p]$ and $[p, K]$ pairs. The findings suggest too that the model predicted scaled values of $v_{\text {dyn }}[\pi, K]$ for $\mathrm{Pb}-\mathrm{Pb}$ collisions are in close agreement with the experimental results. For $[\pi, p]$ and $[p, K]$ pairs, the model predicts relatively higher values for all centrality classes in comparison to those observed in the ALICE experiment

(5) $v_{\text {dyn }}$ values for various collision systems, including ( $p p$ collisions) at LHC energies and for different tunes of HIJING, when plotted against mean charged particle density give a smooth trend. With increasing $\left\langle d N_{\mathrm{ch}} / d \eta\right\rangle$, the values of $v_{\text {dyn }}$ first decrease up to $\left\langle d N_{\mathrm{ch}} / d \eta\right\rangle \sim 100$ and thereafter acquire saturation. The results reported based on ALICE data are also observed to be in accord with the modelbased findings

\section{Data Availability}

The data used in this study are available from the corresponding author upon request.

\section{Conflicts of Interest}

The authors declare that they have no conflicts of interest.

\section{References}

[1] S. A. Voloshin, V. Koch, and H. G. Ritter, "Event-by-event fluctuations in collective quantities," Physical Review C, vol. 60, no. 2, article 024901, 1999.
[2] B. Ali, S. Khan, A. Chandra, and S. Ahmad, "Event-by-event fluctuations clusterization and entropy production in AA collisions at AGS and SPS energies," International Journal of Modern Physics E: Nuclear Physics, vol. 28, no. 3, article 1950018, 2019.

[3] M. Döring and V. Koch, "Event-by-Event uctuations in heavyion collisions," Acta Physica Polonica B, vol. 33, p. 1495, 2002.

[4] L. D. Landau and E. M. Lifscitz, Statistical Physics, Pergamon Press, 1958.

[5] J. L. Albacete, A. Dumitru, and C. Marquet, "The initial state of heavy ion collisions," International Journal of Modern Physics A: Particles and Fields; Gravitation; Cosmology; Nuclear Physics, vol. 28, no. 11, article 1340010, 2013.

[6] M. Luzum and H. Petersen, "Initial state fluctuations and final state correlations in relativistic heavy-ion collisions," Journal of Physics. G, Nuclear and Particle Physics: An Institute of Physics Journal, vol. 41, no. 6, article 063102, 2014.

[7] C. Gale, S. Jeon, and B. Schenke, "Hydrodynamic modeling of heavy-ion collisions," International Journal of Modern Physics E: Nuclear Physics, vol. 28, no. 11, p. 1340011, 2013.

[8] E. Shuryak, "Quark-gluon plasma-new frontiers," Journal of Physics. G, Nuclear and Particle Physics: An Institute of Physics Journal, vol. 35, no. 10, p. 104044, 2008.

[9] H. van Hees, V. Greco, and R. Rapp, "Heavy-quark probes of the quark-gluon plasma and interpretation of recent data taken at the BNL Relativistic Heavy Ion Collider," Phys. Rev. C, vol. 73, no. 3, article 034913, 2006.

[10] S. Ahmad, M. M. Khan, S. Khan, A. Khatun, and M. Irfan, "A study of event-by-event fluctuations in relativistic heavy-ion collisions," International Journal of Modern Physics E: Nuclear Physics, vol. 23, no. 11, article 1450065, 2014.

[11] E. A. De Wolf, I. M. Dremin, and W. Kittel, "Scaling laws for density correlations and fluctuations in multiparticle dynamics," Physics Reports, vol. 270, pp. 1-141, 1996.

[12] R. C. Hwa and C. B. Yang, "Observable properties of quarkhadron phase transition at the Large Hadron Collider," Acta Phys. Polon. B, vol. 48, no. 1, p. 23, 2017.

[13] Y. Xie, G. Chen, J. Wang, Z. H. Liu, and M. J. Wang, "Scaling properties of multiplicity fluctuations in heavy-ion collisions simulated by AMPT model," Nuclear Physics A, vol. 920, pp. 33-44, 2013.

[14] B. Ali, S. Khan, and S. Ahmad, "Contributions of jets in net charge fluctuations from the beam energy scan at RHIC and LHC," Advances in High Energy Physics, vol. 2019, Article ID 6034981, 14 pages, 2019.

[15] S. Ahmad, A. R. Khan, M. Zafar, and M. Irfan, "On multifractality and multifractal specific heat in ion-ion collisions," Chaos, Solitons \& Fractals, vol. 42, no. 1, pp. 538-547, 2009.

[16] R. C. Hwa, "Fractal measures in multiparticle production," Physical Review D, vol. 41, no. 5, pp. 1456-1462, 1990.

[17] S. Khan and S. Ahmad, "Multifractal characteristics of multiparticle production in heavy-ion collisions at SPS energies," International Journal of Modern Physics E: Nuclear Physics, vol. 27, no. 01, article 1850004, 2018.

[18] M. L. Cherry, A. Dabrowska, P. Deines-Jones, P. Holynski, and B. S. Nilsen, "Event-by-event analysis of high multiplicity $\mathrm{Pb}$ (158 GeV/nucleon)-Ag/Br collisions," Acta Physica Polonica B, vol. 29, pp. 2129-2146, 1998.

[19] K. Fialkowski and R. Wit, "Event-by-event cluster analysis of final states from heavy ion collisions," Acta Physica Polonica $B$, vol. 30, p. 2759, 1999. 
[20] S. Ahmad, A. Chandra, A. Kumar et al., "Event-by-event analysis of high-multiplicity events produced in $158 \mathrm{~A}$ $\mathrm{GeV} / \mathrm{c} 208 \mathrm{~Pb}-208 \mathrm{~Pb}$ collisions," EPL (Europhysics Letters), vol. 112, no. 4, p. 42001, 2015.

[21] R. C. Hwa, "Beyond intermittency: erraticity," Acta Physica Polonica B, vol. 27, p. 1789, 1996.

[22] S. Ahmad, M. M. Khan, N. Ahmad, and A. Ahmad, "Erraticity behaviour in relativistic nucleus-nucleus collisions," Journal of Physics. G, Nuclear and Particle Physics: An Institute of Physics Journal, vol. 30, no. 9, pp. 1145-1152, 2004.

[23] S. Ahmad, M. M. Khan, M. M. Khan et al., "Erratic fluctuations in $14.5 \mathrm{~A} \mathrm{GeV/c} 28 \mathrm{Si}-\mathrm{AgBr}$ collisions," (Acta Physica Hungarica A) Heavy Ion Physics, vol. 25, no. 1, pp. 105-115, 2006.

[24] M. Gazdzicki, M. I. Gorenstein, and M. Mackowiak-Pawlowska, "Normalization of strongly intensive quantities," Physical Review C, vol. 88, no. 2, article 024907, 2013.

[25] M. I. Gorenstein and K. Grebieszkow, "Strongly intensive measures for transverse momentum and particle number fluctuations," Physical Review C, vol. 89, no. 3, article 034903, 2014.

[26] K. Grebieszkow and NA61/SHINE Collaboration, "New results on spectra and fluctuations from NA61/SHINE," https://arxiv.org/abs/1904.03165.

[27] B. Sharma, M. M. Aggarwal, N. R. Sahoo, and T. K. Nayak, "Dynamical charge fluctuations in the hadronic medium," Physical Review C, vol. 91, no. 2, article 024909, 2015.

[28] B. Abelev, J. Adam, D. Adamová et al., "Net-charge fluctuations in $\mathrm{Pb}-\mathrm{Pb}$ collisions atsNN=2.76 TeV," Physical Review Letters, vol. 110, no. 15, p. 152301, 2013.

[29] B. I. Abelev, M. M. Aggarwal, Z. Ahammed et al., "Beam-energy and system-size dependence of dynamical net charge fluctuations," Physical Review C, vol. 79, no. 2, article 024906, 2009.

[30] D. K. Mishra, P. Garg, P. K. Netrakanti, L. M. Pant, and A. K. Mohanty, "Experimental results on charge fluctuations in heavy-ion collisions," Advances in High Energy Physics, vol. 2017, Article ID 1453045, 14 pages, 2017.

[31] V. K. Singh, D. K. Mishra, and Z. Ahammed, "Fluctuations of identified particle yields using the $v$ dynvariable at energies available at the BNL Relativistic Heavy Ion Collider," Phys. Rev. C, vol. 101, no. 1, article 014903, 2020.

[32] C. Pruneau, S. Gavin, and S. Voloshin, "Methods for the study of particle production fluctuations," Phys. Rev. C, vol. 66, no. 4, article 044904, 2002.

[33] A. N. Tawfik, I. I. Abou-Salem, A. G. Shalaby, and M. Hanafy, "On dynamical net-charge fluctuations within a hadron resonance gas approach," Advances in High Energy Physics, vol. 2016, Article ID 2475916, 7 pages, 2016.

[34] K. Adcox, S. S. Adler, N. N. Ajitanand et al., "Net charge fluctuations in $\mathrm{Au}+\mathrm{Au}$ interactions at $\mathrm{sNN}=130 \mathrm{GeV}$," Physical Review Letters, vol. 89, no. 8, article 082301, 2002.

[35] P. Christiansen, E. Haslum, and E. Stenlund, "Number-ratio fluctuations in high-energy particle production," Phys. Rev. $C$, vol. 80, no. 3, article 034903, 2009.

[36] S. Jeon and V. Koch, "Charged particle ratio fluctuation as a signal for quark-gluon plasma," Physical Review Letters, vol. 85, no. 10, pp. 2076-2079, 2000.

[37] S. Jeon and V. Koch, "Fluctuations of particle ratios and the abundance of hadronic resonances," Physical Review Letters, vol. 83, no. 26, pp. 5435-5438, 1999.

[38] H. Wang and STAR Collaboration, "Scaling properties and charge dependence of particle ratio fluctuations at RHIC," Central European Journal of Physics, vol. 10, p. 1282, 2012.
[39] V. Koch, A. Majumder, and J. Randrup, "Baryon-strangeness correlations: a diagnostic of strongly interacting matter," Physical Review Letters, vol. 95, no. 18, p. 182301, 2005.

[40] T. Anticic, B. Baatar, J. Bartke et al., "Phase-space dependence of particle-ratio fluctuations in $\mathrm{Pb}+\mathrm{Pb}$ collisions from 20Ato 158A GeV beam energy," Phys. Rev. C, vol. 89, no. 5, p. 054902, 2014.

[41] L. Adamczyk, J. K. Adkins, G. Agakishiev et al., "Energy dependence of $\mathrm{K} \pi, \mathrm{p} \pi$, and $\mathrm{Kp}$ fluctuations in $\mathrm{Au}+\mathrm{Au}$ collisions froms NN=7.7to $200 \mathrm{GeV}$," Phys. Rev. C, vol. 92, no. 2, article 021901, 2015.

[42] T. Tarnowsky, "Measuring dynamical $K / \pi$ and $\mathrm{p} / \pi$ fluctuations in AuAu collisions from the STAR experiment," Physics of Atomic Nuclei, vol. 75, no. 5, pp. 546-549, 2012.

[43] ALICE Collaboration, S. Acharya, D. Adamová et al., "Relative particle yield fluctuations in $\mathrm{Pb}-\mathrm{Pb}$ collisions at $\sqrt{\mathrm{s}_{\mathrm{NN}}}=2.76$ TeV," European Physical Journal C: Particles and Fields, vol. 79, no. 3, p. 236, 2019.

[44] M. Arslandok and ALICE Collaboration, "Event-by-event identified particle ratio fluctuations in $\mathrm{PbPb}$ collisions with alice using the identity method," Nuclear Physics A, vol. 956, p. 870, 2016.

[45] H. Ma, K. Lin, W. L. Qian, and B. Wang, "Centrality dependence of multiplicity fluctuations from a hydrodynamical approach," Advances in High Energy Physics, vol. 2020, Article ID 6504290, 7 pages, 2020.

[46] B. I. Abelev, M. M. Aggarwal, Z. Ahammed et al., "K/ $\pi$ fluctuations at relativistic energies," Physical Review Letters, vol. 103, no. 9, article 092301, 2009.

[47] M. Gyulassy and X. N. Wang, "HIJING 1.0: a Monte Carlo program for parton and particle production in high energy hadronic and nuclear collisions," Computer Physics Communications, vol. 83, no. 2-3, pp. 307-331, 1994.

[48] X. N. Wang and M. Gyulassy, "hijing: a Monte Carlo model for multiple jet production in pp,pA, and AA collisions," Phys. Rev. D, vol. 44, no. 11, pp. 3501-3516, 1991.

[49] T. Sjöstrand and M. van Zijl, "A multiple-interaction model for the event structure in hadron collisions," Phys. Rev. D, vol. 36, no. 7, pp. 2019-2041, 1987.

[50] H. U. Bengtsson and T. Sjöstrand, "The Lund Monte Carlo for hadronic processes - PYTHIA version 4.8," Computer Physics Communications, vol. 46, no. 1, pp. 43-82, 1987.

[51] B. Andersson, G. Gustafson, and B. Nilsson-Almqvist, "A model for low-pT hadronic reactions with generalizations to hadron-nucleus and nucleus-nucleus collisions," Nuclear Physics B, vol. 281, no. 1-2, pp. 289-309, 1987.

[52] B. Nilsson-Almqvist and E. Stenlund, "Interactions between hadrons and nuclei: the Lund Monte Carlo - FRITIOF version 1.6 -," Computer Physics Communications, vol. 43, no. 3, pp. 387-397, 1987.

[53] A. Capella, U. Sukhatme, and J. Tran Thanh van, "Soft multihadron production from partonic structure and fragmentation functions," Zeitschrift für Physik C Particles and Fields, vol. 3, no. 4, pp. 329-337, 1980.

[54] J. Ranft, "Hadron production in hadron-nucleus and nucleusnucleus collisions in the dual Monte Carlo multichain fragmentation model," Phys. Rev. D, vol. 37, no. 7, pp. 18421850, 1988.

[55] J. Ranft, “Transverse energy distributions in nucleus-nucleus collisions in the dual monte carlo multi-chain fragmentation model," Physics Letters B, vol. 188, p. 379, 1987. 
[56] M. Gyulassy and M. Plümer, "Jet quenching in dense matter," Physics Letters B, vol. 243, no. 4, pp. 432-438, 1990.

[57] D. K. Mishra, P. Garg, P. K. Netrakanti, and A. K. Mohanty, "Effect of resonance decay on conserved number fluctuations in a hadron resonance gas model," Physical Review C, vol. 94, no. 1, article 014905, 2016.

[58] R. Nayak, S. Dash, B. K. Nandi, and C. Pruneau, "Modeling of charged kaon and neutral kaon fluctuations as a signature for the production of a disoriented chiral condensate in A-Acollisions," Physical Review C, vol. 101, no. 5, article 054904, 2020.

[59] J. Zaranek, "Measures of charge fluctuations in nuclear collisions," Physical Review C, vol. 66, no. 2, article 024905, 2002.

[60] Q. Liu and T. A. Trainor, "Jet quenching and event-wise mean$\mathrm{p}_{\mathrm{t}}$ fluctuations in central $\mathrm{Au}-\mathrm{Au}$ collisions at $\mathrm{sNN}=200 \mathrm{GeV}$ in Hijing-1.37," Physics Letters B, vol. 567, no. 3-4, pp. 184-188, 2003.

[61] R. Singh, L. Kumar, P. K. Netrakanti, and B. Mohanty, "Selected experimental results from heavy-ion collisions at LHC," Advances in High Energy Physics, vol. 2013, Article ID 761474, 22 pages, 2013.

[62] W. Deng, X. Wang, and R. Xu, "Hadron production in $\mathrm{p}+\mathrm{p}$, $\mathrm{p}+\mathrm{Pb}, \mathrm{Pb}+\mathrm{Pb}$ collisions with the HIJING 2.0 model at energies available at the CERN Large Hadron Collider," Physical Review C, vol. 83, no. 014915, p. 1, 2011.

[63] N. Armesto and C. Pajares, "Central rapidity densities of charged particles at rhic and lhc," International Journal of Modern Physics A: Particles and Fields; Gravitation; Cosmology; Nuclear Physics, vol. 15, no. 14, pp. 2019-2051, 2000.

[64] N. Armesto, "Review of Monte Carlo methods for particle multiplicity evaluation," Journal of Physics Conference Series, vol. 5, pp. 219-229, 2005.

[65] K. Aamodt, ALICE Collaboration, A. Abrahantes Quintana et al., "Centrality dependence of the charged-particle multiplicity density at midrapidity in $\mathrm{Pb}-\mathrm{Pb}$ collisions atsNN=2.76 TeV," Physical Review Letters, vol. 106, no. 3, article 032301, 2011.

[66] J. Adam, D. Adamova, M. M. Aggarwal et al., "Centrality Dependence of the Charged-Particle Multiplicity Density at Midrapidity in $\mathrm{Pb}-\mathrm{Pb}$ Collisions at $\mathrm{sNN}=5.02 \mathrm{TeV}$," Physical Review Letters, vol. 116, pp. 222-302, 2016.

[67] S. Acharya, F. Torales-Acosta, D. Adamová et al., "Centrality and pseudorapidity dependence of the charged-particle multiplicity density in Xe-Xe collisions atsNN=5.44TeV," Physics Letters B, vol. 790, pp. 35-48, 2019.

[68] Indranil Das, CERN-THESIS-2010-261, 2011.

[69] J. Adam, L. Adamczyk, J. R. Adams et al., "Collision-energy dependence of second-order off-diagonal and diagonal cumulants of net-charge, net-proton, and net-kaon multiplicity distributions in Au+Au collisions," Phys. Rev. C, vol. 100, no. 1, article 014902, 2019.

[70] J. T. Balewski, A. Budzanowski, H. Dombrowski et al., "Total cross section of the reaction $\mathrm{pp} \rightarrow \mathrm{pK}+\Lambda$ close to threshold," Physics Letters B, vol. 420, no. 1-2, pp. 211-216, 1998.

[71] V. Koch and T. Schuster, "Energy dependence ofK/ $\pi$ fluctuations in relativistic heavy-ion collisions," Phys. Rev. C, vol. 81, no. 3, article $034910,2010$.

[72] T. Sjöstrand, S. Ask, J. R. Christiansen et al., "An introduction to PYTHIA 8.2," Computer Physics Communications, vol. 191, pp. 159-177, 2015. 